\title{
Memory for Spatial Location: Influences of Environmental Cues and Task Field Rotation
}

\author{
Sylvia Fitting, Douglas H. Wedell, and Gary L. Allen \\ Department of Psychology, University of South Carolina, \\ Columbia, SC 29208 USA \\ fitting@sc.edu
}

\begin{abstract}
We developed a formal theoretical extension of the categoryadjustment model (Huttenlocher, Hedges, \& Duncan, 1991) to incorporate the potential impact of external reference cues in spatial memory. This extension was tested in an experiment in which individuals remembered locations within a circular task field, with different numbers of peripheral cues available. Orientation of the task field was dynamic in the sense that it was rotated on the majority of trials. By modeling the angular bias shown in observers' estimates on unrotated trials, we sought to distinguish between cue-based categories and viewer-based geometric categories as the source of such bias. Results were consistent with our fuzzy boundary extension of the category-adjustment model in which observers generate prototypes based on available reference cues. Both memory accuracy and bias were affected by the number of cues in the task field as predicted.
\end{abstract}

Keywords: spatial memory; bias; category effect; spatial cues; mental rotation.

\section{Memory for Spatial Location}

Upon first consideration, memory for spatial location seems a simple matter. The ease with which a stationary observer can recall where a flash appeared in the night sky, where a "blip" disappeared from a radar screen, or where a familiar person stood in a crowd might suggest a straightforward, uncomplicated process. However, the literature on spatial cognition indicates that, theoretically speaking, spatial memory is a rather complicated phenomenon involving different types of memory, spatial frames of reference, and coding processes.

The purpose of this investigation was to examine how characteristics of a spatial memory task, specifically the availability of environmental cues surrounding the task field and potential changes in task field orientation, influence performance and thus shape our inferences about spatial memory processes. Central to our approach were three considerations. First, we selected a memory task that included a stationary observer remembering a location in a small circular task space. This task provided a straightforward set of requirements, thus providing a clear picture of the influences of environmental cues and task field rotation. Second, we developed a specific quantitative model of the memory coding processes 
that would illustrate clearly the presence and magnitude of the effects of interest. Third, we focused on the memory-based performance of individuals rather than of groups. This case study approach provided a rigorous test of the application of the model while avoiding potential problems arising from averaging across subjects who may employ different strategies. Our aim then was to develop a generally applicable theory-grounded framework for examining spatial processing of environmental cues within a dynamic task field.

\subsection{Types of Spatial Memory and Frame of Reference}

Distinctions between different kinds of spatial memory, frames of reference, and types of memory coding central to each are ubiquitous in the literature (Morris \& Parslow, 2004; Nadel, 1990; Newcombe \& Huttenlocher, 2000). How might these common theoretical distinctions apply to remembering the location of a point in a circular field? One possibility is that an observer could rely on a responselearning mechanism (Nadel, 1990) grounded in an egocentric frame of reference (Klatzky, 1998). Such a mechanism would be consistent with the workings of a perception-action system as posited by Proffitt and his colleagues (Creem \& Proffitt, 1998). Spatial behavior based on this system would be consistent and unbiased by contextual information, although it would be severely disrupted by changes in task demands brought about by field rotation.

A second possibility is that an observer could respond on the basis of a cuelearning mechanism (Nadel, 1990) involving an allocentric object-based frame of reference (Newcombe \& Huttenlocher, 2000). An object-based reference system in this case brings with it some ambiguity. Certainly, a discrete landmark within the circular field would provide the basis for cue-guided responding (Nadel, 1990). However, the circular field itself could possibly be interpreted as an object with intrinsic axes. Past research has demonstrated that observers tend to impose horizontal and vertical axes on a two-dimensional circular field in remembering locations (Huttenlocher, Hedges, \& Duncan, 1991). Memory would be expected to show bias reflecting the implicit or explicit structure of task objects or the task field (Newcombe \& Huttenlocher, 2000). Furthermore, contextual information and task demands would significantly impact performance.

A third possibility is that an observer's memory for location in this task could be the product of a place learning mechanism (O'Keefe \& Nadel, 1978; Tolman, 1948), incorporating an allocentric coordinate frame of reference (Morris \& Parslow, 2004; Nadel, 1990). However, there are different ways in which behavior could be based on a coordinate system. Huttenlocher et al. (1991) suggested that stationary observers tend to impose an implicit polar coordinate system on a circular task field in the absence of visual cues. However, it is also possible to use cue-based axes extending from visible peripheral cues to organize the field, as rats evidently do in the Morris Water Maze task (Morris, Garrud, Rawlins, \& O'Keefe, 1982). In theory, place memory is consistent and unbiased (O'Keefe \& Nadel, 1978), although its reliability is strictly dependent upon stable spatial relations among environmental objects. In fact, there has 
been relatively little empirical work examining the effects of task demands and contextual information on place memory per se.

Closely related to the concept of different types of spatial memory is the theoretical distinction between different spatial coding processes (Allen \& Haun, 2004; Kosslyn et al., 1989). A well specified and empirically verified distinction of this type involves categorical and fine-grain coding (Huttenlocher et al., 1991). Categorical coding is posited as the robust product of a relatively rapid process in which location is remembered in terms of its being within a particular categorical region of the response space. A central or salient location within this category acts as a prototype, biasing remembered locations toward it. In the specific task of a stationary observer remembering a location with a circular field, observers appear to impose implicit horizontal and vertical axes on the circle, thus creating quadrants that function as categories (Huttenlocher et al., 1991), with centrally located points within these quadrants serving as prototypes. Remembered locations are "pulled" toward these prototypes.

Fine-grain coding is posited as the product of the somewhat more timeconsuming process of remembering a location in terms of a geometric coordinate system imposed upon the response field. Fine-grain coding yields metric accuracy and less susceptibility to bias. Previous research has shown that stationary observers remembering a location within a circular field encode fine-grain information with respect to a polar coordinate system imposed on the response field (Huttenlocher et al., 1991). Fine-grain memory may also be more fragile and hence decay more rapidly than categorical information, resulting in a greater reliance on categorical coding with increased memory demands (Haun, Allen, \& Wedell, 2005).

\subsection{Spatial Memory as a Multifaceted Phenomenon}

The preceding considerations suggest that a stationary observer remembering a location in a circular field could rely on an egocentric, an allocentric-intrinsic, or an allocentric-coordinate frame of reference and presumably could switch from one to the other in the face of task demands. Memory in this task could involve categorical coding, fine-grain coding, or both. Accordingly, it seems advisable to approach spatial memory as a multifaceted phenomenon involving an array of cognitive tools that can be flexibly enacted in response to task situations.

Based on this view, we sought to establish how well-documented findings across different tasks could be integrated into the current task. With respect to cue availability, it is well established that observers tend to impose implicit quadrants on a circular task space to code spatial location categorically (Huttenlocher et al., 1991), and it is well established that animals use cues peripheral to a circular response area to organize their memory of that space (Morris et al., 1982). However, it is not clear how observers in our memory task would use peripheral cues outside the circular response field. They could ignore them, relying instead on the implicit category structure imposed on the circular field, or they could use them as spatial prototypes for categorical coding. 
With respect to field rotation, it is well established that observers can track spatial location when response fields are rotated relative to the observers' initial view. Thus, it was assumed that they could accommodate the rotation of the circular field used in this work, given an adequate cue for orientation. However, it is not known how the possibility of task field rotation per se affects such coding (as when memory trials requiring rotation are intermixed with trials requiring no rotation).

We addressed these issues by developing an extension of Huttenlocher et al.'s (1991) category-adjustment model, which features a combination of finegrain and categorical coding. First, we formally examined how categories reflecting viewer-based horizontal and vertical axes would bias memory and then contrasted that prospective outcome with how categories reflecting peripheral cues in the task field would bias memory. Subsequently, we conducted an experiment designed to show which of the two versions of categorical organization was a better predictor of actual memory bias. In the next section, we present our modeling framework in some detail.

\section{Fine-Grain and Categorical Coding in Place Memory}

\subsection{The Category-Adjustment Model}

Our model builds on the category-adjustment model of Huttenlocher et al. (1991), according to which spatial location is coded at two levels, fine-grain and categorical. Each of these sources involves unbiased error. Accordingly, fine-grain information is centered on the actual location, whereas categorical information is centered on the category prototype. Bias in memory arises from combining information from these two sources to produce an estimate. Although this combination produces a predictable pattern of bias, it also results in an overall reduction in error (Huttenlocher et al., 1991).

Because bias in estimation is central to our investigation, we present a series of formal models that will be used to generate testable predictions. According to the category-adjustment model, the expected value of the response in an estimation task, $\mathrm{E}[R]$, can be characterized as a weighted average of fine-grain and categorical information described by the following equation:

$$
E[R]=\lambda \mu+(1-\lambda) p
$$

where $\mu$ is the mean of the distribution of fine-grain memory values for the object, assumed to be unbiased and hence equated with the true location of the object. Similarly, $p$ is the mean of the distribution of prototype locations for the relevant category. The parameter $\lambda$, which varies from 0 to 1 , represents the relative weight of the fine-grain information. Bias is determined by subtracting the actual value from the response, and thus the expected bias is characterized by the following equation:

$$
E[\text { Bias }]=E[R]-\mu=\lambda \mu+(1-\lambda) p-\mu .
$$




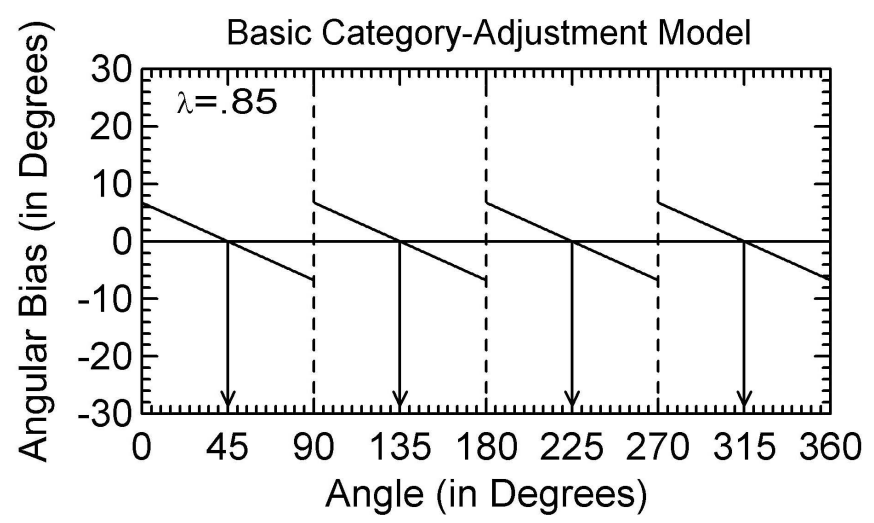

Fig. 1. Category-adjustment model of Equation (2) applied with boundaries fixed along the axes (i.e., at $0^{\circ}, 90^{\circ}, 180^{\circ}$, and $270^{\circ}$ ) and prototypes at the midpoint of each quadrant (i.e., at $45^{\circ}, 135^{\circ}, 225^{\circ}$, and $315^{\circ}$ ).

Within Huttenlocher's framework the weighting of fine-grain memory depends on the relative uncertainty of the fine-grain memory value and the prototype memory value. In general, the greater the uncertainty concerning fine-grain information, the less it is weighed (i.e., $\lambda$ decreases). In Equations (1) and (2), $\lambda$ is a constant, reflecting an assumption that uncertainty does not vary across locations within a category.

Figure 1 illustrates the model's prediction for remembered locations within a circular field. Following Huttenlocher et al. (1991), we impose a polar coordinate system that locates the dot in terms of its angle and radial distance from the center. In our examples, we focus on the angular component of the prediction, although similar predictions can be made concerning radial distance. As with Huttenlocher et al. (1991), we assume an implicit categorical structure that divides the circle into four quadrants along vertical and horizontal axes. As shown in Figure 1, estimates are unbiased when stimuli are located at the category protypes. Conversely, bias is maximized near the boundaries of each quadrant, where the deviation from the prototype is maximal.

One shortcoming with the model of Equation (2) is that it does not account for nonlinear bias that can appear in the data. For example, Haun et al. (2005) found that bias decreased near extreme angles of $0^{\circ}$ and $90^{\circ}$ that were the category boundaries in their azimuth and incline estimation tasks. They argued that this debiasing near the borders could be explained in terms of greater fine-grain memory discrimination, consistent with the bowing effect observed in perceptual judgments (Luce, Nosofsky, Green, \& Smith, 1982). Increased discrimination for fine-grain information near the border would be reflected in corresponding higher weighting of $\lambda$. They used a simple quadratic function to capture this relationship as follows:

$$
\lambda=a+b(\mu-p)^{2},
$$



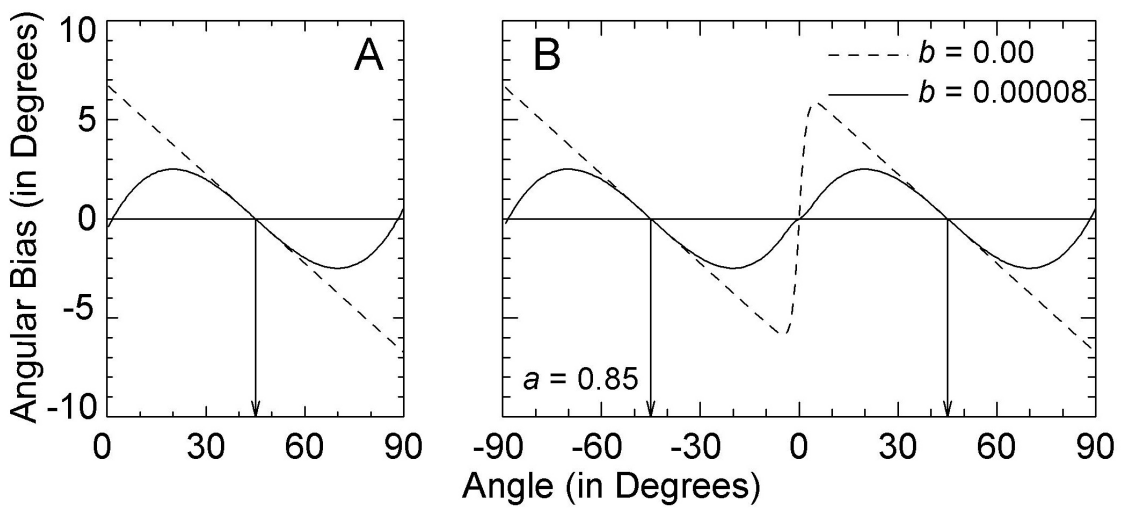

Fig. 2. Model prediction of bias under conditions of constant weighting (dashed line) of fine-grain information $(b=0)$ or a curvilinear relationship (solid line) between weight and angle $(b>0)$, with $a$ representing baseline fine- grain weighting. Panel A: Application of Equations (2) and (3) to estimation within a single quadrant assuming a prototype value of $45^{\circ}$. Panel B: Application of Equations (2) through (4) to estimation across adjacent quadrants assuming prototypes at $-45^{\circ}$ and $45^{\circ}$.

where $a$ represents the baseline weighting of fine-grain memory and $b$ indexes the increment to this value as the stimulus deviates from the prototype and thus appoaches a boundary or border. In the Haun et al. model (2005), the expression for $\lambda$ from Equation (3) was substituted into Equation (2) to model the increase in weighting of fine-grain memory values near the border. The pattern of bias produced from this model is presented in Panel A of Figure 2.

In Haun et al.'s (2005) Experiment 2, motor estimates of azimuth across a range of angles that spanned two quadrants were not well fit by the combination of Equations (2) and (3), primarily because estimates near the border appeared to be influenced by both prototypes. To accommodate this possibility, Haun et al. (2005) proposed a fuzzy boundary version of the category-adjustment model in which stimuli near the border beween two categories were sometimes misclassified and hence recruited the wrong category prototype. For the two category case involving a single boundary, the probability of retrieving a given prototype can be described as a logistic function of the difference between the actual angle and the category boundary location as follows:

$$
\operatorname{Pr}\left(p_{1} \mid \mu\right)=\frac{1}{1+\exp (-c(\mu-t))}
$$

where $p_{1}$ represents one of two prototypes, $c$ is a sensitivity or scaling parameter, and $t$ is the threshold or boundary dividing the two categories. Within this framework, the probability of retrieving the other prototype is simply the complementary probability. The pattern of bias produced from a model that combines Equations (2), (3) and (4) is presented in Panel B of Figure 2. 


\subsection{Further Developments}

Potentially, the category-adjustment model is widely applicable. Thus far, it has been applied in its basic form to memory for locations in two-dimensional fields (Huttenlocher et al., 1991; Newcombe \& Huttenlocher, 2000) and in modified form to memory for incline and azimuth (Haun et al., 2005). To apply the fuzzy boundary model of Haun et al. to the location memory task, however, requires some modification. One problem is that the task implies the use of four categories rather than two so that the logistic probability function cannot be used. To meet this problem, we propose a probability recruitment function that is based on the relative similarity of the stimulus angle to the midpoints of all the possible categories. A frequently applied similarity function is the exponential decay function (Nosofsky, 1986; Shepard, 1987), in which similarity falls off very rapidly with increased distance. We first apply this similarity function to the fixed four quadrant case. Accordingly, the probability of prototype recruitment can be described as follows:

$$
\operatorname{Pr}\left(p_{j} \mid \mu\right)=\frac{\exp \left(-c\left|\mu-.5\left(t_{M I N, j}+t_{M A X, j}\right)\right|\right)}{\sum \exp \left(-c\left|\mu-.5\left(t_{M I N, k}+t_{M A X, k}\right)\right|\right)}
$$

where similarity is calculated relative to the midpoint of the category, using the average of the lower boundary, $t_{M I N}$, and the upper boundary, $t_{M A X}$. By using the midpoints of boundaries, Equation (5) represents a fixed threshold (fuzzy boundary) model. Note that because of the polar coordinate system, angles that are in the first and fourth quadrants are incorrectly seen as distant from each other (i.e., $1^{\circ}$ and $359^{\circ}$ are seen as $358^{\circ}$ apart rather than $2^{\circ}$ apart). Thus, to properly apply this model we include two additional midpoints corresponding to the lowest valued midpoint plus $360^{\circ}$ (i.e. $45^{\circ}+360^{\circ}=405^{\circ}$ ) along with the highest valued midpoint minus $360^{\circ}$ (i.e. $315^{\circ}-360^{\circ}=-45^{\circ}$ ). These virtual midpoints are necessary to allow the model to recruit the quadrant 1 prototype for quandrant 4 angles, and vice versa. In short, recruitment of prototypes from either clockwise or counter-clockwise rotation requires the addition of these "virtual" category midpoints.

Figure 3 shows the predicted pattern of bias from a model that combines Equations (2) and (5) for the location memory task. Note that as with the model of Figure 1, fixed boundaries are assumed at vertical and horizontal axes. While bias at first increases as the angle moves away from the prototype location, it later decreases as the boundary is approached. This phenomenon occurs because near the boundary the angle is increasingly likely to recruit the prototype from the adjacent category, and hence bias is added in the opposite direction. Although the prototype locations are shown at the midpoints of the categories, the model combining Equations (2) and (5) requires only that the prototype lie somewhere within the category boundaries.

The focus of our study is on the potential influence of cues peripheral to the task field on estimation bias. The most straight-forward extension of the fuzzy boundary category adjusment model is to assume that the cues serve as 


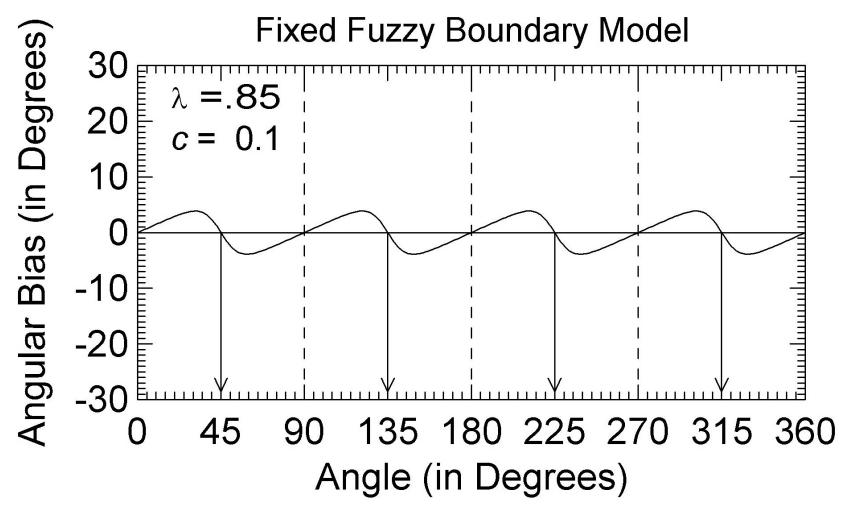

Fig. 3. Fuzzy boundary model of Equations (2) and (5) with fixed boundaries at $0^{\circ}$, $90^{\circ}, 180^{\circ}$, and $270^{\circ}$ dividing the circle into four quadrants (prototypes located at $45^{\circ}, 135^{\circ}, 225^{\circ}$, and $315^{\circ}$ ). Bias is reduced near the boundaries due to recruitment of prototypes from adjacent categories. Note $\lambda=$ weighting of fine-grain information and $c=$ sensitivity parameter.

prototypes. Note that this assumption means that we can no longer use the fixed boundaries described by viewer-based vertical and horizontal axes. Instead, we infer boundaries by assuming they fall at equal distances from the category prototypes. Accordingly, we alter the prototype recruitment equation to be based on the similarity of the stimulus to the prototype rather than to the midpoint of the category as follows:

$$
\operatorname{Pr}\left(p_{j} \mid \mu\right)=\frac{\exp \left(-c\left|\mu-p_{j}\right|\right)}{\sum \exp \left(-c\left|\mu-p_{k}\right|\right)} .
$$

Once again we include "virtual" prototypes for the lowest and highest categories so that recruitment may be conducted in a clockwise or counter-clockwise fashion. Note that even when there is only one prototype, there will be a "virtual" boundary created by the inclusion of these virtual prototypes. For example, consider the case in which the cue and, accordingly, the category prototype is located at $305^{\circ}$. Virtual prototypes will be created at $665^{\circ}$ and $-55^{\circ}$. Given that similarity falls off exponentially, the virtual prototype at $665^{\circ}$ will have no impact. However, the prototype at $-55^{\circ}$ will have an impact. Indeed, angles in quadrant $1\left(0^{\circ}\right.$ to $\left.90^{\circ}\right)$ will recruit this virtual prototype and hence exhibit a negative bias. The point halfway between the prototype at $305^{\circ}$ and at $-55^{\circ}$ (i.e., at $125^{\circ}$ ) will then represent the virtual boundary in this single prototype case.

Panel A of Figure 4 describes the predictions of this cue-based fuzzy boundary version of the category-adjustment model for the one prototype case (with the prototype at $305^{\circ}$ ). As shown, the bias function crosses $0^{\circ}$ at two points, once at the prototype value of $305^{\circ}$ and once at the virtual boundary value of $125^{\circ}$. This striking pattern of bias is quite different from the usual pattern shown in Figures 1 and 3 and thus should be easily detected in observers' behavior. 


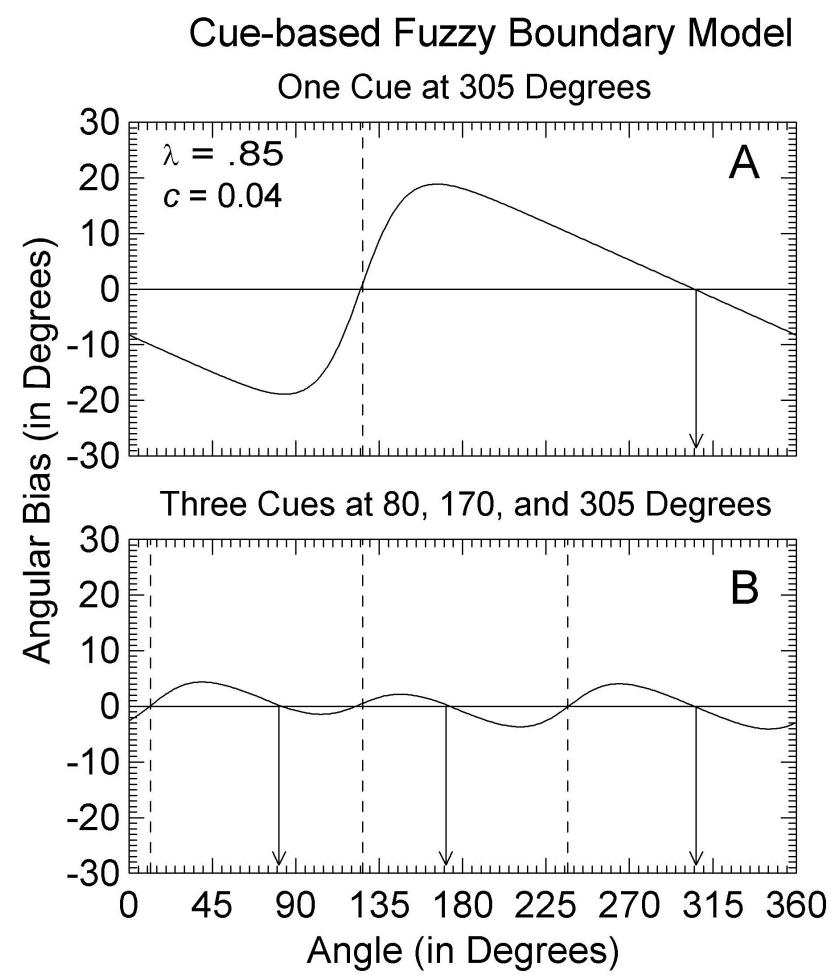

Fig. 4. Predictions of the fuzzy boundary model of Equations (1) and (6), with prototypes equated with cue location, probabilistic recruitment of prototypes across categories, and inferred boundaries located halfway between adjacent prototypes. Panel A: One cue case with the prototype at $305^{\circ}$ and the boundary at $125^{\circ}$. Panel B: Three prototypes located at $80^{\circ}, 170^{\circ}$, and $305^{\circ}$, with inferred boundaries at $237.5^{\circ}, 12.5^{\circ}$ and $125^{\circ}$. Note $\lambda=$ weighting of fine-grain information and $c=$ sensitivity parameter.

Panel B of Figure 4 describes the predictions of the cue-based fuzzy boundary model for the three-cue case with prototypes located at $p=80^{\circ}, p=170^{\circ}$, and $p=305^{\circ}$. Once again two virtual prototypes are added, one at $p=440^{\circ}$ and the other at $p=-55^{\circ}$. These prototypes produce inferred boundaries at $237.5^{\circ}, 12.5^{\circ}$ and $125^{\circ}$. The bias pattern generated by three prototypes (Panel B) is thereby highly distinguishable from the pattern produced by one prototype (Panel A). It is also quite distinct from the quadrant-based four-category case shown in Figures 1 and 3 .

In summary, the developments here show how the category-adjustment model can be modified to account for fuzzy boundaries and cue-based prototypes in a task requiring memory for location in a circular field. The patterns of bias have been shown to be quite different, depending on the number and location of cues. We may therefore pose the question of whether actual observers show bias in memory reflecting cue-based categories. 


\section{Effects of Cues and Possible Field Rotation on Spatial Coding}

\subsection{Rationale}

In a previous study, Fitting (2005) tested different versions of the categoryadjustment model in a memory for location task similar to the one conducted by Huttenlocher et al. (1991). In this study, number of peripheral cues was varied, and the task field was in a fixed orientation. Results from all conditions were consistent with the modified category-adjustment model shown in Figure 3. Four prototypes located near the middle in each of four quadrants were implicitly imposed by observers, with quadrant boundaries determined by vertical and horizontal axes. The lack of any significant interactions with cue condition provided evidence for a cue-independent spatial coding process within a static task field. Observers simply ignored the available external reference cues.

The lack of cue effects in this previous experiment prompted us to consider the possible influence of a dynamic context generated by adding a preponderance of trials in which the task field was rotated. Thus, we designed a study in which the number of potential reference cues was varied and there was the possibility of task field rotation. In this paper, we consider only the unrotated trials because they afford the use of either coding strategy, that is, categories based on the vertical and horizontal axes of the viewer (Fitting, 2005; Huttenlocher et al., 1991) or categories based on cue locations.

\subsection{Method}

In our task, participants attempted to reproduce the location of 32 dots in a circular field presented on a two-dimensional computer display, with 16 dots located at a short radius of 92 pixels and 16 dots located at a long radius of 168 pixels. In each of these two sets, four different angles $\left(3^{\circ}, 25^{\circ}, 43^{\circ}\right.$, and $\left.75^{\circ}\right)$ were presented in each of the four quadrants (based on cardinal directions). In order to create a dynamic task field setting, four equally occurring rotations were included in this task (rotations of $0^{\circ}, 30^{\circ}, 90^{\circ}$, or $160^{\circ}$ ). Participants were tested under two conditions of cue availability. In the one-cue condition, an external cue appeared at $305^{\circ}$ just outside the circular field. In the three-cue condition, unique external cues appeared at $80^{\circ}, 170^{\circ}$, and $305^{\circ}$ just outside the circular field. Thus, each cue set consisted of 128 trials (4 rotations of 32 dot locations). Note that rotation consisted of moving the locations of the external cues around the field by the prescribed angle.

All materials and instructions were presented on computers with 15-in. (38$\mathrm{cm})$ monitors at a resolution of $640 \times 480$ pixels. The circular region was identical to the white background and separated by a 20 pixel black circle (radius $=212$ pixels). A red dot, 5 pixel in radius, was the target.

Participants were administered a series of training trials with and without rotation. These example trials were followed by the two actual test sets with 128 different dot locations. Each dot appeared on screen for $1 \mathrm{~s}$, and then was 
covered by a dynamic checkerboard mask for $1.5 \mathrm{~s}$ followed by a blank circle. After the first set, there was a 3-min break. In the second set, the cue condition was changed. Subjects moved a cross-hairs cursor to the remembered location using a mouse and clicked a mouse button to record the response (measured in pixel units on the screen).

The dependent variable we focused on was 'angular bias', calculated by subtracting the angle of the actual location from the angle of the reproduced location. A negative value indicated a clockwise bias, whereas a positive value indicated a counter-clockwise angular bias.

\section{$3.3 \quad$ Results}

This paper presents results for angular bias scores of two individual participants whose pattern of responding was representative of the group data. Our casestudy approach is powerful because it focuses on a within-subject design and shows how participants' estimates depend on available reference cues. Subject A experienced the one-cue condition first and the three-cue condition second; whereas Subject B experienced these conditions in the reverse order. One outlying data point from Subject A was deleted as it was displaced beyond $40^{\circ}$.

Figure 5 presents the data for Subjects A and B under the one-cue and threecue conditions. A cursory glance at the pattern of data clearly supports two contentions. First, the pattern of data is inconsistent with the simple categoryadjustment model that assumes fixed boundaries along the geometric axes of the circle. Thus, these results differ dramatically from those of Fitting (2005), in which there was no prospect of field rotation. Second, the patterns of bias for one and three cues strongly resemble the corresponding patterns shown in Figure 4, as predicted by the fuzzy boundary model with cue-determined prototypes.

To provide a more formal analysis, we fit different models to the data and used the change in proportion of variance explained $\left(R^{2}\right)$ as a main criterion for assessing the model fit. The angular bias scores for the two subjects were fit separately to the original category-adjustment model by Huttenlocher et al. (1991) and the different versions of the fuzzy boundary model presented here.

The fit of the category-adjustment model of Equation (2) is presented in Table 1 for both subjects in the one-cue and three-cue condition, along with estimated parameter values. One feature of the Huttenlocher et al. model (1991) is the assumption of one prototype in each of the four quadrants. This assumption implies four downward sloping functions that cross the $0^{\circ}$ bias line in the graphs at the locations of the prototype in each of the four quadrants. This clearly is not the case for the one-cue condition in which the bias functions appear to cross $0^{\circ}$ bias line only twice. It also does not appear to be the case for the three-cue functions. Another feature of the original model is that it assumes a linear bias function without taking into account the possibility of fuzzy boundaries. Our data are not consistent with that outcome. Also, the original model predicts little or no bias, as fine-grain weighting values are close to 1.0 (as shown in Table 1). Inconsistent with this value of $\lambda$ are the large observed deviations from the $0^{\circ}$ bias line, especially for the one-cue condition. Finally, the inferred value 

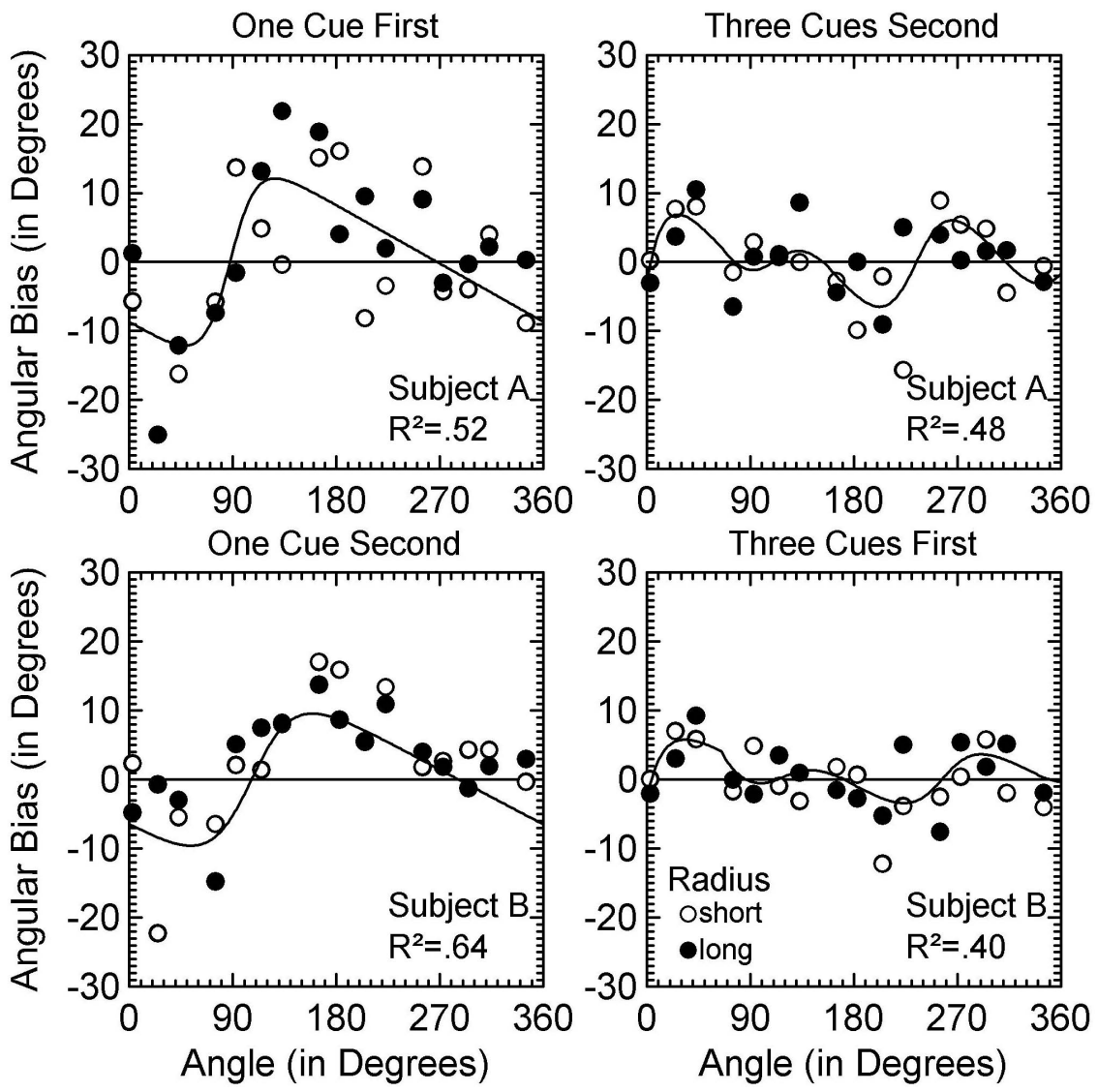

Fig. 5. Empirical angular bias scores plotted separately for one-cue and three-cue conditions along with model prediction functions. Panel A: Subject A. Panel B: Subject B.

of the $\lambda$ parameter exceeds 1.0 for both subjects in the one-cue condition, a value that contradicts the spirit of the model. In conclusion, the category-adjustment model with geometrically determined boundaries provides a poor fit to these data.

The cue-based fuzzy boundary model of Equations (2) and (6) overcomes the shortcoming of the four-quadrant version of the model. Versions of the fuzzy boundary model were generated by successively freeing parameter values and using the iterative nonlinear regression procedure within SYSTAT (Wilkinson, 1989), with a least squared error criterion. Parameters were freed only if doing so led to a significant increment in $R^{2}$. The estimated parameter values of the best-fit versions of the model are presented in Table 2 and the predictions from these models are plotted in Figure 5. Even though the proportions of explained variance are not high in all four cases, there is clear evidence that the subjects 
Table 1. Parameters for the Fit of the 5-Parameter Category-Adjustment Model of Equation (2)

\begin{tabular}{llllllll}
\hline Subject Cue condition & \multicolumn{4}{c}{ Parameter } & $R^{2}$ \\
\hline & & $p_{1}$ & $p_{2}$ & $p_{3}$ & $p_{4}$ & $\lambda$ & \\
A & One cue first & $80^{\circ}$ & $123^{\circ}$ & $200^{\circ}$ & $340^{\circ}$ & 1.117 & 0.252 \\
B & One cue second & $80^{\circ}$ & $98^{\circ}$ & $183^{\circ}$ & $315^{\circ}$ & 1.114 & 0.315 \\
& & & & & & & \\
A & Three cues second $78^{\circ}$ & $169^{\circ}$ & $183^{\circ}$ & $350^{\circ}$ & 0.991 & 0.053 \\
B & Three cues first & $83^{\circ}$ & $145^{\circ}$ & $183^{\circ}$ & $345^{\circ}$ & 0.950 & 0.269 \\
\hline
\end{tabular}

Note: $p=$ prototype value and $\lambda=$ weight of fine-grain memory.

Table 2. Parameters for the Fit of Fuzzy Boundary Model of Equations (2) and (6)

\begin{tabular}{llcccccc}
\hline Subject Cue condition & \multicolumn{5}{c}{ Parameter } & $R^{2}$ \\
\hline & & $p_{1}$ & $p_{2}$ & $p_{3}$ & $\lambda$ & $c$ & \\
A & One cue first & $268^{\circ}$ & - & - & 0.906 & 0.043 & 0.523 \\
B & One cue second & $286^{\circ}$ & - & - & 0.911 & 0.027 & 0.635 \\
& & & & & & & \\
A & Three cues second & $66^{\circ}$ & $156^{\circ}$ & $310^{\circ}$ & 0.807 & 0.035 & 0.478 \\
B & Three cues first & $64^{\circ}$ & $171^{\circ}$ & $344^{\circ}$ & 0.913 & 0.035 & 0.399 \\
\hline
\end{tabular}

Note: $p=$ prototype value, $\lambda=$ weight of fine-grain memory, $c=$ sensitivity parameter

are influenced by the available reference cues. In the one-cue condition, both individuals are less biased near the location of the external cue, with the estimated prototype location being near the actual external cue location of $305^{\circ}$. Furthermore, freeing parameter values to include more prototypes did not significantly increase the fit of the model. In contrast to the one-cue condition, both observers evidently relied on three prototypes when three cues were available. Again the locations of the three prototypes estimated by the model are near the actual values of the external reference cues, $80^{\circ}, 170^{\circ}$, and $305^{\circ}$. Here, the model fitting procedure clearly rejected models based on one or two prototypes, and the inclusion of a fourth prototype did not significantly increment the fit of the model.

In order to test for changes in accuracy across cue conditions, within-subject analyses of variance (ANOVA's) were conducted on the absolute values of angular bias scores, with cue condition as a repeated measure and using the three-way interaction term as an error term. Both participants were significantly less accurate when just one cue was available, $F(1,8)=26.17, p<.001$ and $F(1,9)$ $=8.31, p=.018$, for Subjects A and B respectively. These results support the basic notion behind the category-adjustment model that including categorical information from multiple categories can reduce overall error. 


\section{Conclusions}

Our results and those of related studies indicate that spatial memory, even in tasks involving nothing more than specifying a location seen moments earlier, is a multifaceted phenomenon that can involve a number of different processes in response to task demands and available information. Previous studies have established that memory for location involves coding two types of information, fine-grain and categorical (Allen \& Haun, 2004; Huttenlocher et al., 1991). Finegrain information is metrically veridical and based on a coordinate frame of reference (for example, polar coordinates). In contrast, categorical information is based on properties of the task field. Prototypes within categories draw remembered locations toward them, resulting in an unmistakable pattern of bias.

When observers remember locations within a circular field in the absence of peripheral cues, their memory is biased by implicit categories imposed on the circle itself (Huttenlocher et al., 1991). Specifically, observers tend to impose viewer-based horizontal and vertical axes on the circle, with the resulting quadrants acting as categories and an interior point within each quadrant serving as a category prototype. The presence of peripheral cues exerts no influence when field rotation is not a possibility (Fitting, 2005). In other words, observers tend to ignore the cues and impose viewer-based horizontal and vertical axes. The result is the same four-category structure that influences memory in the cue-free version of the task.

When the task includes both rotated and unrotated trials, however, the unrotated trials, which pose the same prima facie task demands as trials in the Fitting (2005) study with a static field, show a very different categorical structure. On these trials, the cues that are necessary for orientation during rotated trials are also used on unrotated trials. In short, the categorical structure imposed on the task field is cue-based, with the number of cues dictating the number of categories and corresponding prototypes.

In addressing the influence of environmental cues on categorical coding of location information, we developed a fuzzy boundary extension of Huttenlocher et al.'s (1991) category-adjustment model. Our aim was not only to develop a way of addressing this issue in its inherent complexity, but also to offer a more general theoretical tool for investigating the interplay between fine-grain and categorical coding of spatial relations in a variety of tasks. For example, recent research showed that this type of model provides a good account of memory for incline and azimuth, with categories based on implicit quadrants imposed on the response field (Haun et al., 2005). Our current findings suggest that this outcome may be limited to response fields that lack cues. Such cues in the visual environment may afford the viewer a different categorical structure.

Another potential application of our approach involves classic place learning tasks, such as the Morris Water Maze. In the current study, we employed a task that was comparable in some ways to the Morris Water Maze, but clearly there were differences. Navigation is involved in the Morris Water Maze, but not in our task. The Morris Water Maze typically involves a single place to be learned; our task involved hundreds of trials with different locations to be 
remembered. Thus, the results from our experiment are more suggestive than conclusive when addressing the question of whether categorical bias is present in animal and human behavior in this classic place learning paradigm. We hope to obtain more conclusive evidence with humans using two different approaches. One approach involves a place learning task performed by humans in an actual arena in which navigation is required. In a study using this approach, Fitting and Allen (2004) found some evidence that participants distorted remembered locations toward implicit axes extending from peripheral cues, a finding consistent with cue-based categorical coding. A different approach to the same issue involves a computerized version of the water maze task that requires navigation. We are designing studies using this approach in which participants guide a virtual rat in discovering and subsequently navigating to a safe platform in the maze. As with the location memory task we used in the current study, reliance on cue-based categorical coding should be revealed in memory bias.

Another question that needs further investigation is the applicability of the model in a large-scale space. How do the spatial memory processes proposed by the fuzzy boundary model come into play when location memory is tested in a large-scale space? This question raises interesting issues regarding the process by which observers parse the environment into categorical regions, the role of environmental geometry and peripheral cues in this parsing process, and the role of human information-processing limitations in cue selection and use.

Given our view of spatial memory as a multifaceted phenomenon involving an array of cognitive tools, we suspect that different contextual and task constraints may lead individuals to apply cue-based categories in large-scale tasks in ways similar to those found in the present small-scale study. From this perspective, a key issue for future research is the determination of conditions leading to the use of different spatial representations and cognitive tools across spatial environments.

\section{References}

Allen, G. L, \& Haun, D. B. M. (2004). Proximity and precision in spatial memory. In G. Allen (Ed.), Human Spatial Memory: Remembering where (pp. 41-61). Mahwah, NJ: Lawrence Erlbaum Associates.

Creem, S. H., \& Proffitt, D. R. (1998). Two memories for geographical slant: Separation and interdependence of action and awareness. Psychonomic Bulletin and Review, 5, 22-36.

Fitting, S. \& Allen, G. L. (November, 2004). Remembering places in spaces: Memory load, retention interval and environmental cues. Presented at the 45th annual meeting of the Psychonomic Society. Minneapolis, MN.

Fitting, S. (2005) Memory for spatial location: Cue effects as a function of field rotation. Master's Thesis, Department of Psychology, University of South Carolina, Columbia, SC.

Haun, D. B. M., Allen, G. L., \& Wedell, D. H. (2005). Bias in spatial memory: A categorical endorsement. Acta Psychologica, 118, 149-170.

Huttenlocher, J., Hedges, L. V., \& Duncan, S. (1991). Categories and particulars: Prototype effects in estimating spatial location. Psychological Review, 98, 352-376. 
Klatzky, R. (1998). Allocentric and Egocentric Spatial Representations: Denitions, Distinctions, and Interconnections. In C. Freksa, C. Habel, \& K. Wender (Eds.). Spatial cognition: An interdisciplinary approach to representation and processing of spatial knowledge (pp. 1-17). Berlin: Springer-Verlag.

Kosslyn, S. M., Koenig, O., Barrett, A., Cave, C. B., Tang, J., \& Gabrieli, J. D. (1989). Evidence for two types of spatial representations: Hemispheric specialization for categorical and coordinate relations. Journal of Experimental Psychology: Human Perception and Performance, 15, 723-735.

Luce, R. D., Nosofsky, R. M., Green, D. M., \& Smith, A. F. (1982). The bow and sequential effects in absolute identification. Perception and Psychophysics, 32, 397-408.

Morris, R. G. M., Garrud, P., Rawlins, J. N. P., \& O’Keefe, J. (1982). Place navigation impaired in rats with hippocampal lesions. Nature, 297, 681-683.

Morris, R. G., \& Parslow, D. M. (2004). Neurocognitive components of spatial memory. In G. Allen (Ed.). Human Spatial Memory: Remembering where (pp. 217-247). Mahwah, NJ: Lawrence Erlbaum Associates.

Nadel, L. (1990). Varieties of spatial cognition: Psychobiological considerations. In A. Diamond (Ed.), Annals of the New York Academy of Sciences (Vol. 608; pp. 613636). New York: New York Academy of Sciences.

Newcombe, N. S., \& Huttenlocher, J. (2000). Making space: The developmental of spatial representation and spatial reasoning. Cambridge, MA: MIT Press.

Nosofsky, R. M. (1986). Attention, similarity, and identification-categorization relationship. Journal of Experimental Psychology: General, 115, 39-61.

O'Keefe, J., \& Nadel, L. (1978). The hippocampus as a cognitive map. Oxford: Clarendon Press.

Shepard, R. N. (1987). Toward a universal law of generalization for psychological science. Science, 237, 1317-1323.

Tolman, E. C. (1948). Cognitive maps in rats and men. Psychological Review, 55, 189208.

Wilkinson, L. (1989). SYSTAT: The system for statistics. Evanston, IL: SYSTAT, Inc. 